\title{
Evaluation of the reliability of two field hockey specific sprint and dribble tests in young field hockey players
}

\author{
K A P M Lemmink, M T Elferink-Gemser, C Visscher
}

Br J Sports Med 2004;38:138-142. doi: 10.1136/bjsm.2002.001446

See end of article for authors' affiliations

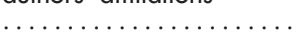

Correspondence to: Dr K A P M Lemmink, University of Groningen, Institute of Human Movement Sciences, A. Deusinglaan 1, Groningen 9713 AV The Netherlands: k.a.p.m.lemmink@ ppsw.rug.nl

Accepted 26 March 2003

\begin{abstract}
Objectives: To determine the reliability of two field hockey specific tests: the shuttle sprint and dribble test (ShuttleSDT) and the slalom sprint and dribble test (SlalomSDT).

Methods: The shuttle sprint and dribble performances of 22 young male and 12 young female field hockey players were assessed on two occasions within 4 weeks. Twenty one young female field hockey players took part in the slalom sprint and dribble test twice in a 4 week period.

The ShuttleSDT required the players to perform three $30 \mathrm{~m}$ shuttle sprints while carrying a hockey stick alternated with short periods of rest and, after a 5 minute rest, three $30 \mathrm{~m}$ shuttle sprints alternated with rest while dribbling a hockey ball. The SlalomSDT required the players to run a slalom course and, after a 5 minute rest, to dribble the same slalom with a hockey ball.

Results: There were no differences in mean time scores between the two test sessions. The mean differences were small when compared with the means of both test sessions. With the exception of the slalom sprint time, zero lay within the $95 \%$ confidence interval of the mean differences indicating that no bias existed between the two measurements. With the exception of delta shuttle time (0.79), all intraclass correlation coefficient values for the ShuttleSDT, met the criterion for reliability of 0.80 . Intraclass correlation coefficient values for SlalomSDT were 0.91 for slalom sprint time, 0.78 for slalom dribble time, and 0.80 for delta slalom time.

Conclusions: ShuttleSDT and the SlalomSDT are reliable measures of sprint and dribble performances of young field hockey players.
\end{abstract}

$\mathrm{R}$ ecent developments in field hockey, such as the artificial playing surface, new stick material, and the interchange rule have increased the number of physiological and technical demands made on field hockey players at all levels, but in particular at the elite level. Competitive field hockey matches place heavy aerobic demands on players and require them to expend energy at relatively high levels. ${ }^{12}$ High intensity activities such as cruising, sprinting, and activities in which the player is directly involved with the ball (for example, dribbling) have been shown to represent between $17.5-30 \%$ of the competition time, ${ }^{3}$ and are considered critical to the outcome of the game. Furthermore, in field hockey, high and low intensity activities alternate by a ratio ranging from about $1: 4$ to $1: 8 .^{3}$ Consequently, as well as maximal performance on individual high intensity activities, the ability to produce high intensity efforts is crucial for top level field hockey players.

Field hockey is a multiple high intensity activity sport with a multidirectional nature. The ability to change direction rapidly while maintaining balance without loss of speedthat is, agility-is therefore an important physical component necessary for successful performance in field hockey. Elite field hockey players also need high level technical skills such as being able to dribble without losing running speed. For a technically good player, dribbling is essentially an automatic process, and the better players distinguish themselves by their running speed while dribbling the ball. ${ }^{4}$

Coaches, trainers, and players are continually searching for effective methods of identifying and developing those characteristics in a player that may enhance performance. There are a variety of field tests with which to measure the physiological and technical characteristics of players in team games like soccer, rugby, and handball. However, there was no single test to measure both physiological and technical characteristics in field hockey players and for this reason we developed two tests specifically to measure these characteristics. Based on tests for repeated sprint ability ${ }^{5-12}$ and dribbling skills of field hockey players ${ }^{4}$ and soccer players ${ }^{13}{ }^{14}$ we developed the field hockey specific shuttle sprint and dribble test (ShuttleSDT) to measure shuttle sprint and dribble performance. Based on tests for agility ${ }^{15}$ and dribbling skills of field hockey players ${ }^{4}$ and soccer players, ${ }^{13}{ }^{14}$ the field hockey specific slalom sprint and dribble test (SlalomSDT) was developed to measure slalom sprint and dribble performance.

It is vital that the ShuttleSDT and the SlalomSDT provide reliable information. A reliable test must perform consistently. In other words, if an individual whose ability or skill has not changed is tested twice with a completely reliable measuring device, both scores will be identical. ${ }^{16}$ The aim of this study was therefore to determine the reliability of the ShuttleSDT and the SlalomSDT in young elite field hockey players.

\section{MATERIALS AND METHODS \\ Subjects}

A total of 34 young male $(n=22)$ and female $(n=12)$ field hockey players participated in the reliability study of the field hockey specific shuttle sprint and dribble test (ShuttleSDT). The mean age of the boys was 15.5 (SD 1.79) years, and of the girls 13.8 (SD 1.03) years. Twenty one young female field hockey players whose mean age was 13.5 (SD 1.25) years volunteered to take part in the reliability study of the field hockey specific slalom sprint and dribble test (SlalomSDT).

After being informed about the study procedure, the subjects gave their verbal consent to participation. The group averaged two training sessions and one match per week.

Abbreviations: ICC, intraclass correlation coefficient 


\section{Procedures}

To examine the reliability of the ShuttleSDT and the SlalomSDT, two trials were conducted within a period of 2-4 weeks and during the subjects' normal training hours, varying between 16:30 and 20:00. On day one and two the average temperature during testing was 4.3 and $3.7^{\circ} \mathrm{C}$ respectively, humidity was $86-98 \%$ on both days and wind conditions (no high winds) were comparable. The tests were conducted on a synthetic pitch surface laid on a sandy field with subjects wearing their normal playing footwear. The subjects were only given feedback on their performance after completing all the tests.

\section{Shuttle sprint and dribble test (ShuttleSDT)}

The ShuttleSDT was developed to measure field hockey specific shuttle sprint and dribble performance. This study used a modified version of the Interval Sprint Test protocol first introduced by Lemmink et al. ${ }^{11}$ This was originally performed by soccer players outdoors on a grass surface. Our modifications involved an electronic timing system and slight modifications of the sprint distances, three instead of 10 sprints, and the use of a hockey stick and a hockey ball. The protocol consisted of three maximal sprints of $32 \mathrm{~m}$ while carrying a hockey stick and three maximal sprints of $32 \mathrm{~m}$ while dribbling a hockey ball. Each $32 \mathrm{~m}$ sprint included a $6 \mathrm{~m}$ and a $10 \mathrm{~m}$ shuttle sprint. Timing procedures (timing gates), meant that the initial and final metres of the sprint were not timed, so data are based on $30 \mathrm{~m}$ distances (fig 1 ).

The subject began the test standing with both feet behind line A (marked with two cones $2 \mathrm{~m}$ apart). On an auditory signal after a 5 second countdown, the subject sprinted $6 \mathrm{~m}$ to line $\mathrm{B}$ (marked with two cones,) touched the line with one foot and returned to line A, again touching the line with one foot. The subject then sprinted $10 \mathrm{~m}$ to line C (marked with two cones), touched the line with one foot and returned to finish over line A. The subject then tapered down from the sprint, turned and walked back slowly to line A, there waiting for the 5 second countdown and the auditory signal to start the second sprint. The second sprint started exactly 20 seconds after the start of the first sprint. After the third sprint the subject was allowed 5 minutes recovery time, during which he/she walked. The recovery walk was timed so that the subject had returned to line A 10-20 seconds before the start of the dribbling portion of the test. The protocol of the dribbling portion was identical to the sprinting portion, except that the subject was now dribbling a hockey ball.

Timing data were measured by means of photocell gates (Eraton BV, Weert, The Netherlands) placed at $1.05 \mathrm{~m}$ above ground (approximately at hip height) and at $1.0 \mathrm{~m}$ behind line A. The photocells were linked to an electronic timer with an accuracy of 0.01 seconds. The following variables were noted and recorded:

- sprint times = individual sprint times

- dribble times $=$ individual dribble times

- peak sprint time $=$ fastest sprint time

- peak dribble time $=$ fastest dribble time

- total sprint time = total sprint time of the three sprints

- total dribble time $=$ total dribble time of the three dribbles

- delta shuttle time = difference between the total dribble time and the total sprint time.

\section{Slalom sprint and dribble test (SlalomSDT)}

Based on tests for agility and dribbling skills, the field hockey specific slalom sprint and dribble test (SlalomSDT) was developed to measure field hockey specific slalom sprint and dribble performance. The protocol consisted of a maximal

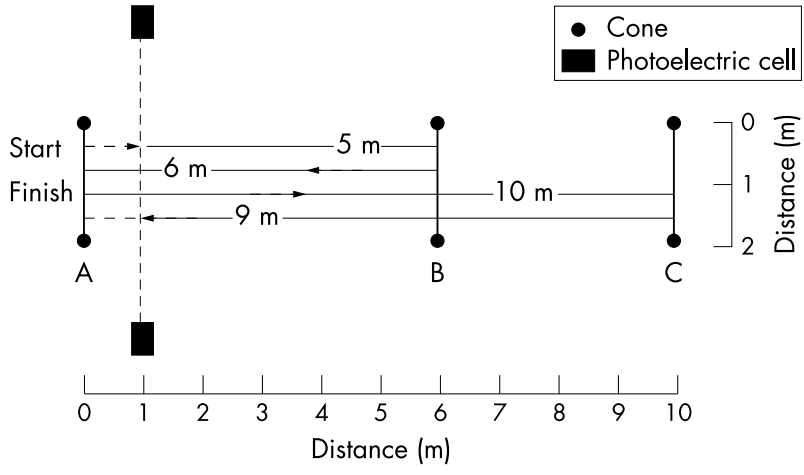

Figure 1 Course of the shuttle sprint and dribble test (ShuttleSDT).

slalom sprint of $30 \mathrm{~m}$ while carrying a hockey stick and a maximal slalom dribble of $30 \mathrm{~m}$ while dribbling a hockey ball. Twelve cones were placed in a zigzag pattern (fig 2). Start and finish lines (A and B) were marked by two cones.

The subject began the test with both feet behind line A; then, upon an auditory signal after a 5 second countdown, the subject ran with a hockey stick around the 12 cones finishing over line B. After the run the subject was allowed 5 minutes for recovery, during which he/she walked slowly. The total distance of the course was $29.07 \mathrm{~m}$. The recovery walk was timed so that the subject had returned to line A 1020 seconds before the start of the next portion. The protocol of the dribbling portion was identical to the sprinting portion, except that the subject was now dribbling a hockey ball. If the subject lost control of the ball-that is, if the subject was more than approximately $2 \mathrm{~m}$ away from the cones, the test was repeated. Timing data were measured using a stopwatch. Slalom sprint time, slalom dribble time, and the difference between the slalom times of the dribble and sprint (delta slalom time) were noted and recorded accurately to within 0.01 seconds.

\section{Statistical analysis}

The ShuttleSDT data are expressed as mean (standard deviation, SD). To determine the relation between the times measured, a correlation matrix was calculated for the test scores at $t_{1}$. A three way (time $\times$ sprint/dribble $\times$ test session) analysis of variance with repeated measures was used to determine differences in times of each sprint/dribble. A Scheffé post hoc test was used to identify specific differences when the main effects were significant.

To determine the reliability of the ShuttleSDT, the data of both boys and the girls were analysed together. ShuttleSDT reliability analysis was carried out on the peak and total times of the sprints and dribbles and on the delta shuttle time. The mean difference between the test results on both days was set as a measure of absolute reliability. If zero lay within the $95 \%$ confidence interval (CI) of the mean difference, we concluded that no bias existed between the measurements. ${ }^{17-19}$

To determine relative reliability, we used a one way analysis of variance (ANOVA) to calculate intraclass correlation coefficients (ICCs) of repeated interval scale measures. ${ }^{16}{ }^{19}{ }^{20}$ Intraclass correlation coefficients were determined for the peak and total sprint and dribble times and for the delta shuttle time. Ninety five per cent confidence intervals were determined for all of the ICCs. ${ }^{19}$ As a general rule, an intraclass correlation coefficient over 0.90 is considered to be high, between $0.80-0.90$ moderate, and below 0.80 to be insufficient for physiological field tests. ${ }^{21}$ Baumgartner and Jackson ${ }^{16}$ stated that ICCs of a minimum of 0.80 are acceptable for physical measures. 


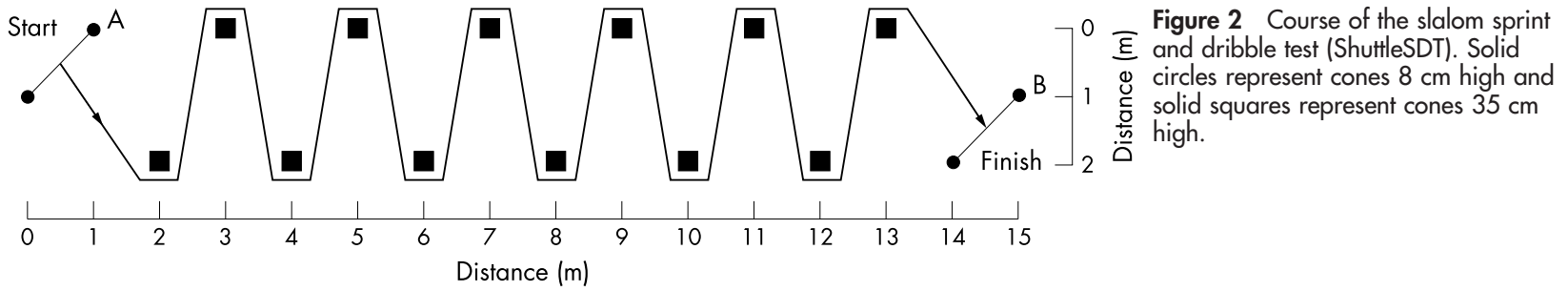

Table 1 Intercorrelations (Pearson correlation coefficients) between the sprint (S) and dribble (D) times, peak sprint and dribble times, total sprint and dribble times, and the calculated delta shuttle time of the ShuttleSDT at $t_{1}(n=34)$. All times are expressed in seconds

\begin{tabular}{|c|c|c|c|c|c|c|c|c|c|c|}
\hline & S2 & S3 & $S_{\text {peak }}$ & $S_{\text {total }}$ & D1 & D2 & D3 & $D_{\text {peak }}$ & $D_{\text {total }}$ & Delta \\
\hline $\begin{array}{l}\text { S1 } \\
\text { S2 } \\
\text { S3 } \\
S_{\text {peak }} \\
S_{\text {total }} \\
\text { D1 } \\
\text { D2 } \\
\text { D3 } \\
D_{\text {peak }} \\
D_{\text {total }} \\
\text { Delta }\end{array}$ & $0.79^{*}$ & $\begin{array}{l}0.80^{*} \\
0.78^{*}\end{array}$ & $\begin{array}{l}0.94^{*} \\
0.87^{*} \\
0.83^{*}\end{array}$ & $\begin{array}{l}0.93^{*} \\
0.93^{*} \\
0.92^{*} \\
0.95^{*}\end{array}$ & $\begin{array}{l}0.22 \\
0.30 \\
0.35 \\
0.24 \\
0.31\end{array}$ & $\begin{array}{l}0.48^{*} \\
0.53^{*} \\
0.60^{*} \\
0.52^{*} \\
0.58^{*} \\
0.75^{*}\end{array}$ & $\begin{array}{l}0.28 \\
0.45^{\star} \\
0.44^{*} \\
0.29 \\
0.43 \\
0.76^{*} \\
0.76^{*}\end{array}$ & $\begin{array}{l}0.35 \\
0.43 \\
0.47^{*} \\
0.38 \\
0.45^{*} \\
0.93^{*} \\
0.84^{*} \\
0.85^{*}\end{array}$ & $\begin{array}{l}0.35 \\
0.47^{*} \\
0.50^{*} \\
0.38 \\
0.48^{*} \\
0.91^{*} \\
0.91^{*} \\
0.93^{*} \\
0.96^{*}\end{array}$ & $\begin{array}{r}-0.15 \\
-0.02 \\
0.03 \\
-0.13 \\
-0.05 \\
0.85^{*} \\
0.69^{*} \\
0.81^{*} \\
0.82^{*} \\
0.86^{*}\end{array}$ \\
\hline
\end{tabular}

The SlalomSDT data are expressed as mean (standard deviation, SD). To determine the relation between the times measured, a correlation matrix was calculated for the test scores at $t_{1}$. A two way (sprint/dribble $\times$ test session) analysis of variance with repeated measures was used to determine the differences in time of the slalom sprint and slalom dribble.

To determine reliability, analysis of variance was performed on the test scores of the SlalomSDT. For absolute reliability the mean difference with a $95 \%$ CI between the testing days was calculated. ${ }^{17-19}$ Intraclass correlation coefficients were calculated with a 95\% CI for the slalom times of the sprint and dribble and the calculated delta slalom time to determine relative reliability.

\section{RESULTS}

The correlation matrix of the ShuttleSDT showed strong correlations between the time scores of the sprinting and dribbling portions individually (table 1). Weak correlations existed between the time scores of the sprints and the dribbles. The delta shuttle time was strongly related to the time scores of the dribbling portion of the ShuttleSDT but not to the time scores of the sprinting portion. SlalomSDT correlations showed a weak relation between the slalom sprint and dribble times $(r=0.24)$. As in the ShuttleSDT, the delta slalom time was strongly correlated with the slalom time of dribbling a hockey ball $(r=0.90)$ but not with the slalom sprint time $(r=-0.21)$.

There were significant differences between the shuttle sprint and dribble times at both test sessions $(p=0.00)$ (fig 3 ). There were no differences in time scores between the test sessions $(p=0.98)$ (fig 3$)$. The mean time of each of the three sprints and dribbles on the ShuttleSDT increased significantly at both tests sessions $(p=0.00)$ (table 2 ). Post hoc analysis showed that each of the three sprint and dribble times was significantly different from the others $(p<0.05)$. For the SlalomSDT, there were significant differences between the sprint and dribble time at both test sessions but not between test sessions $(p=0.09)$.
Table 2 shows the mean (SD) of all sprint and dribble times at the first and second test session $\left(t_{1}\right.$ and $\left.t_{2}\right)$ of the ShuttleSDT and the SlalomSDT, and the mean difference, standard error, and $95 \%$ CI of the mean difference to determine absolute reliability. ${ }^{19}$ In general, the values of the mean differences between the first and second test sessions were small when compared with the means of the two test sessions. With the exception of the slalom sprint time, zero lay within the $95 \% \mathrm{CI}$, which indicates reasonable agreement between the two testing days.

Intraclass correlation coefficient values to assess the relative reliability of the ShuttleSDT ranged from 0.71 for the second sprint time to 0.91 for the peak dribble time (table 3). After excluding the separate sprint and dribble times of the ShuttleSDT, all ICC values (with the exception of

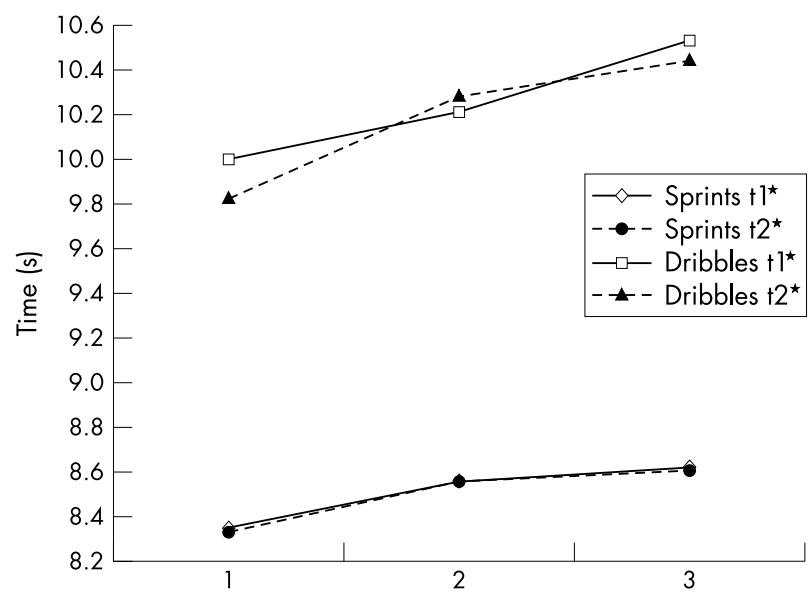

Figure 3 Group data for the sprint and dribble times of the Shuttle Sprint and Dribble Test (ShuttleSDT) at the first and second test sessions $\left(t_{1}\right.$ and $\left.t_{2}\right)$. Means and standard deviations of each data point are presented in table 2. *Significant difference between the first, second, and third sprints and dribbles $(p<0.05)$. 
Table 2 Results of the Bland and Altman method for absolute reliability of the ShuttleSDT $(n=34)$ and the SlalomSDT $(n=21)$

\begin{tabular}{|c|c|c|c|c|c|}
\hline & Mean $t_{1}$ (SD) & Mean $t_{2}$ (SD) & Mean d (SD) & SE of $d$ & $95 \% \mathrm{Cl}$ \\
\hline \multicolumn{6}{|l|}{ ShuttleSDT } \\
\hline Sprint 1 & $8.35(0.525)$ & $8.33(0.579)$ & $-0.026(0.446)$ & 0.076 & -0.182 to 0.129 \\
\hline Sprint 2 & 8.55 (0.567) & $8.55(0.632)$ & $-0.007(0.570)$ & 0.098 & -0.206 to 0.192 \\
\hline Sprint 3 & $8.62(0.522)$ & $8.61(0.664)$ & $-0.010(0.507)$ & 0.087 & -0.187 to 0.167 \\
\hline Peak sprint time & $8.28(0.511)$ & $8.29(0.563)$ & $0.010(0.434)$ & 0.075 & -0.141 to 0.162 \\
\hline Total sprint time & 25.52 (1.495) & $25.48(1.817)$ & $-0.043(1.366)$ & 0.234 & -0.520 to 0.434 \\
\hline Dribble 1 & 10.00 (0.997) & $9.83(1.045)$ & $-0.173(0.737)$ & 0.126 & -0.430 to 0.084 \\
\hline Dribble 2 & $10.21(0.964)$ & $10.29(1.115)$ & $0.077(0.965)$ & 0.165 & -0.260 to 0.413 \\
\hline Dribble 3 & $10.53(1.186)$ & $10.45(1.368)$ & $-0.076(1.098)$ & 0.188 & -0.459 to 0.307 \\
\hline Peak dribble time & $9.79(0.829)$ & $9.66(0.887)$ & $-0.135(0.487)$ & 0.083 & -0.305 to 0.035 \\
\hline Total dribble time & $30.74(2.882)$ & 30.57 (3.093) & $-0.173(1.912)$ & 0.328 & -0.840 to 0.494 \\
\hline Delta shuttle time & $5.22(2.539)$ & $5.09(2.137)$ & $-0.130(1.962)$ & 0.337 & -0.814 to 0.555 \\
\hline \multicolumn{6}{|l|}{ SlalomSDT } \\
\hline Slalom sprint time & $16.36(0.751)$ & $16.15(0.736)$ & $-0.207(0.419)$ & 0.091 & -0.398 to -0.016 \\
\hline Slalom dribble time & 20.93 (1.689) & $20.56(1.513)$ & $-0.366(1.366)$ & 0.298 & -0.988 to 0.256 \\
\hline Delta slalom time & 4.57 (1.679) & $4.41(1.774)$ & $-0.159(1.403)$ & 0.306 & -0.797 to 0.480 \\
\hline
\end{tabular}

the delta shuttle time (0.79)) met the criterion of 0.80 for reliability.

Intraclass correlation coefficient values to assess the SlalomSDTs relative reliability were 0.91 for the slalom sprint time, 0.78 for the slalom dribble time, and 0.80 for the delta slalom time (table 3 ). With the exception of the slalom dribble time, the ICC values met the reliability criterion of 0.80 .

\section{DISCUSSION}

Using repeated sprint ability and agility tests and taking into account the multidirectional and technical nature of field hockey, we developed two field tests to determine shuttle sprint and shuttle dribble performance. Both tests are practical for use on a regular basis as they can be administered easily and are popular with the players. Low correlations and significant differences in mean sprint and dribble times on both tests confirmed our expectation that different physical abilities were being measured. The course, duration, and repetitive nature of the ShuttleSDT mean that the test is probably of more importance for defenders and midfielders. Conversely, the course of the SlalomSDT makes the test more relevant to forwards.

Table 3 Intraclass correlation coefficients for relative reliability of the ShuttleSDT $(n=34)$ and the SlalomSDT $(n=21)$

\begin{tabular}{lll}
\hline & ICC & $95 \% \mathrm{Cl}$ \\
\hline ShuttleSDT & 0.81 & 0.61 to 0.90 \\
Sprint 1 (s) & 0.71 & 0.42 to 0.86 \\
Sprint 2 (s) & 0.78 & 0.56 to 0.89 \\
Sprint 3 (s) & 0.81 & 0.61 to 0.90 \\
Peak sprint time (s) & 0.80 & 0.59 to 0.90 \\
Total sprint time (s) & 0.85 & 0.70 to 0.93 \\
Dribble 1 (s) & 0.73 & 0.45 to 0.86 \\
Dribble 2 (s) & 0.77 & 0.55 to 0.89 \\
Dribble 3 (s) & 0.91 & 0.82 to 0.96 \\
Peak dribble time (s) & 0.89 & 0.77 to 0.94 \\
Total dribble time (s) & 0.79 & 0.58 to 0.89 \\
Delta shuttle time (s) & & \\
SlalomSDT & 0.91 & 0.78 to 0.97 \\
$\quad$ Slalom sprint time (s) & 0.78 & 0.45 to 0.91 \\
Slalom dribble time (s) & 0.80 & 0.51 to 0.92 \\
$\quad$ Delta slalom time (s) & & \\
\hline ICC, intraclass correlation coefficient; Cl, confidence interval.
\end{tabular}

Although several authors use other measures (for example, Pearson's correlation, 95\% limits of agreement, coefficient of repeatability, and coefficient of variation) mean difference, standard errors, 95\% CI of the mean differences, and ICC values have all recently been reported as being most appropriate and clear in determining reliability. ${ }^{19}$ In our reliability data, the $95 \%$ CIs for the mean differences between the test days can be interpreted as a minimum difference between the results of individuals that indicate a real change in performance level. This indicates the accuracy of the test in monitoring changes over time.

We have no reason to expect that reliability of field testing is influenced by the subjects' youth or sex. Other characteristics such as heterogeneity, motivation to do well, and learning capabilities are assumed to be factors that affect reliability in a positive way. ${ }^{16}$ Our subjects were a homogeneous group, very motivated, and with above average learning capabilities as they all played field hockey at the highest regional level. Environmental conditions do influence field testing. Therefore, ambient temperature, humidity, and wind conditions were all documented. There were only minor differences in environmental conditions during the test sessions. Furthermore, tests were conducted on the same artificial grass surface with players wearing their normal playing footwear.

The measurements with the most robust relative reliability were the peak dribble time in the ShuttleSDT and the slalom sprint time in the SlalomSDT (ICC $=0.91$ ). As mentioned earlier, an intraclass correlation coefficient of over 0.90 is considered to be high, between $0.80-0.90$ moderate, and below 0.80 insufficient for physiological field tests. ${ }^{21}$ Based on these criteria, it is reasonable to suggest that the peak sprint and dribble times and the total sprint and dribble times of the ShuttleSDT have an acceptable relative reliability. The delta shuttle time has insufficient reliability $($ ICC $=0.79)$. The absolute reliability data of the ShuttleSDT showed reasonable agreement between both testing sessions.

Reliability ICC values of the SlalomSDT suggest that the slalom sprint and the delta slalom time have acceptable relative reliability. The slalom dribble time has insufficient reliability (ICC $=0.78$ ) and should therefore be used with caution. The reliability of a test depends on many factors, for instance the nature of a test. ${ }^{16}$ Scores may not be stable if subjects have not had experience of or practice at the test before being measured. In tests requiring skill, such as the dribble performance in the ShuttleSDT and the SlalomSDT, 


\section{Take home message}

The ShuttleSDT and the SlalomSDT are sport specific field tests to measure sprint and dribble performances of field hockey players. The tests showed reasonable reliability and can help coaches and trainers assess young athletic talent, differentiate between players, and monitor changes over time.

the learning effect may have influenced the reliability of the scores. This corresponds with the trend of faster times on the dribble performance on the ShuttleSDT and SlalomSDT seen at $t_{2}$. However, only the mean difference and $95 \%$ CI for the slalom sprint time on the SlalomSDT between the first and second testing sessions indicated a bias and, therefore, a lack of absolute reliability. Subjects needed less time for the slalom sprint course at $t_{2}$, indicating that there might have been a learning effect. However, only a series of trials can lead to a more definite conclusion about this.

The reliability coefficients of this study are in line with those obtained when evaluating other field tests in adult field hockey players and other subjects. A study of a 5 m multiple shuttle test in 23 female field hockey players to determine players' match related fitness reported a range of coefficients from ICC $=0.74$ to $0.98 .{ }^{9}$ Fitzsimons et $a l^{10}$ reported correlation coefficients of 0.75 to 0.94 for a running test of repeated sprint ability in 15 male field hockey players. Pauole et $a l^{15}$ reported an intraclass reliability coefficient of 0.98 for a test of agility ( $\mathrm{T}$ test) in college aged men and women. Finally, Baker et $a l^{6}$ reported a Pearson correlation coefficient of 0.86 for a repeated maximal shuttle run test in 10 male subjects.

In summary, coaches and trainers can use the field tests examined in this study, as the demands of the test are important ones for field hockey performance (alternation of high and low intensity activities, agility, speed, and technical skills). It is also a practical test to use on a regular basis because it can be administered easily. The ShuttleSDT and the SlalomSDT can help coaches and trainers to assess young athletic talent, diagnose specific weaknesses, provide information for the development of individualised training programmes, and assess changes in physical characteristics as a result of a training cycle. This study has shown that the absolute and relative reliability of the ShuttleSDT and the SlalomSDT are satisfactory.

\section{ACKNOWLEDGEMENTS}

The authors wish to express their sincere appreciation to Mieke Richart and Inge Scheek for their assistance in this research project.

\section{Authors' affiliations}

K A P M Lemmink, M T Elferink-Gemser, C Visscher, Institute of Human Movement Sciences, University of Groningen, the Netherlands

\section{REFERENCES}

1 Boyle PM, Mahoney CA, Wallace WFM. The competitive demands of elite male field hockey. J Sports Med Phys Fitness 1994;34:235-41.

2 Reilly T, Borrie A. Physiology applied to field hockey. Sports Med 1992;14:10-26.

3 Lothian F, Farrally M. A time-motion analysis of women's hockey. J Hum Movement Studies 1994;26:255-65.

4 Reilly T, Bretherton S. Multivariate analysis of fitness of female field hockey players. In: Day JAP, ed. Perspectives in kinanthropometry. Champaign, IL: Human Kinetics, 1986:135-42.

5 Aziz AR, Chia M, Teh KC. The relationship between maximal oxygen uptake and repeated sprint performance indices in field hockey and soccer players. J Sports Med Phys Fitness 2000;40: 195-200.

6 Baker J, Ramsbottom R, Hazeldine R. Maximal shuttle running over $40 \mathrm{~m}$ as a measure of anaerobic performance. Br J Sports Med 1993;27:228-32.

7 Bangsbo J. The physiology of soccer-with special reference to intense intermittent exercise. Acta Physiol Scand 1994;15(suppl 619):1-156.

8 Bishop D, Spencer M, Duffield R, et al. The validity of a repeated sprint ability test. J Sci Med Sport 2001;4:19-29.

9 Boddington MK, Lambert MI, St Clair Gibson A, et al. Reliability of a $5 \mathrm{~m}$ multiple shuttle test. J Sports Sci 2001;19:223-8.

10 Fitzsimons $M$, Dawson $B$, Ward D, et al. Cycling and running tests of repeated sprint ability. Austr J Sci Med Sport 1993;25:82-7.

11 Lemmink KAPM, Dolleman G, Verheijen R, et al. Interval Sprint Test en Interval Shuttle Run Test-betouwbaarheid en discriminerend vermogen van twee nieuwe voetbaltests. Geneeskunde en sport 2000;33:39-48 [English abstract].

12 Wragg CB, Maxwell NS, Doust JH. Evaluation of the reliability and validity of a soccer-specific field test of repeated sprint ability. Eur J Appl Physiol 2000;83:77-83.

13 Reilly T, Holmes M. A preliminary analysis of selected soccer skills. Phys Educ Rev 1983;6:64-71

14 Rossum JHA van, Wijbenga D. Soccer skills technique tests for youth players: construction and implications. In: Reilly T, Clarys J, Stibbe A, eds. Science and football II. London: E \& FN Spon, 1993:313-18.

15 Pauole K, Madole K, Garhammer J, et al. Reliability and validity of the T-test as a measure of agility, leg power, and leg speed in college-aged men and women. J Strength Cond Res 2000;14:443-50.

16 Baumgarter TA, Jackson AS. Measurement for evaluation in physical education and exercise science. Boston, MA: WCB-McGraw-Hill, 6th edition, 1999.

17 Altman DG, Gardner MJ, eds. Calculating confidence intervals for means and their differences. In: Statistics with confidence. London: BMJ Books, 1989:20-7.

18 Bland JM, Altman DG. Statistical methods for assessing agreement between two methods of clinical measurement. Lancet 1986;1:307-10.

19 Rankin G, Stokes M. Reliability of assessment tools in rehabilitation: an illustration of appropriate statistical analyses. Clin Rehab 1998;12:187-99.

20 Baumgartner TA. Norm-referenced measurement: reliability. In: Safrit MJ, Woods TM, eds. Measurement concepts in physical education and exercise science. Champaign, IL: Human Kinetics, 1989:45-72.

21 Vincent WJ. Statistics in kinesiology. Champaign, IL: Human Kinetics, 1995. 